\title{
Extending a User Involvement Tool with Virtual and Augmented Reality
}

\author{
Ciprian Florea ${ }^{1}$, Paula Alavesa ${ }^{1}$, Leena Arhippainen ${ }^{1}, 2$, Matti Pouke ${ }^{1}$, Weiping Huang ${ }^{1}$, Lotta Haukipuro ${ }^{3}$, Satu \\ Väinämö ${ }^{4}$, Arttu Niemelä5, Marta Cortés Orduña ${ }^{1}$, Minna A Pakanen ${ }^{6}$, Timo Ojala ${ }^{1}$ \\ ${ }^{1}$ Center for Ubiquitous Computing, University of Oulu ${ }^{2}$ INTERACT Research Unit, University of Oulu ${ }^{3} 5$ GenZ project, University of Oulu \\ ${ }^{4}$ Centre for health and technology, University of Oulu ${ }^{5}$ Trä Group Oy, 00160 Helsinki, Finland ${ }^{6}$ Socio-Technical Design, Department of \\ Engineering, Inge Lehmanns Gade 10, 8000 Aarhus, Denmark
}

\begin{abstract}
Living labs are environments for acquiring user feedback on new products and services. Virtual environments can complement living labs by providing dynamic immersive setup for depicting change.

This paper describes implementation of Virtual and Augmented Reality clients as an extension to a user involvement tool for an existing living lab. We conducted a user experience study with 14 participants to compare the clients. According to our study, the virtual reality client was experienced as innovative, easy to use, entertaining and fun. Whereas the augmented reality client was perceived playful and empowering.
\end{abstract}

Keywords: living lab, user involvement, user experience, virtual reality, augmented reality.

Index Terms: H.5.1 [Information Interfaces and Presentation] Multimedia Information Systems-Artificial, augmented, and virtual realities

\section{INTRODUCTION}

The living lab approach has become a popular way of involving users into innovation and evaluation processes of products and services. Unlike mere field trials or user testing, a living lab approach involves users in all stages of the product development lifecycle $[5,7]$. The approach is relevant especially when studying user experience (UX) before, during and after the use of products or services. Current Web applications can support innovation creation activities in living labs, as these applications are inherently social. They facilitate user participation, content creation and sharing, conversation, cooperation and collaboration. For example, a social media-based living lab approach can integrate technologies of social networks with the living labs concept [4]. Virtual environments have successfully been utilized in participatory studies involving visualizations of change $[5,8,7]$. Both virtual reality (VR) and augmented reality (AR) offer even more immersive mechanisms for using VEs in visualization. These technologies are at the forefront or technological advancements and have also recently made their way into consumer products. Utilizing VR in living labs has potential to largely benefit current user communities.

\footnotetext{
1-4[firstname.lastname]@oulu.fi

5arttu.niemela@trad.fi

${ }^{6}$ mpakanen@eng.au.dk
}

\section{WebVR and AR in User Involvement Process}

As described by McNeese [5] and Pierson and Lieven [7], exposure to graspable simulations and reconfigurable prototypes i.e. test worlds are key elements of living labs. Through VR, users can navigate and observe a VE in a similar way they would in a real world. In VR, the user can inhabit or embody a test world, that then demands a higher presence than mere images. When VR is made available through Web, it can also inherit the benefits of Web applications. While WebVR is already being used for professional visualization and entertainment, applications involving user participation are currently scarce [2]. Like VR, or WebVR, AR can be utilized as a visualization tool that leverages the social aspects of Web. While less immersive than VR, mobile AR can provide free exploration of joined realities. The users can simultaneously experience both the digital and the physical environments which can be beneficial when gathering feedback from, for example, planned changes in the physical environment $[1,3]$. In addition, AR technology is more easily available, as most smart phones and tablets support AR content [1].

\section{Prototype System}

Our prototype system allows the generation of questionnaires for multiple platforms, i.e. Web, mobile, VR and AR. This is managed through a single administrative web tool (the Admin Tool). Besides regular web-based questionnaires, the author of a questionnaire can utilize the Admin Tool to generate location-based questionnaires, as well as questionnaires utilizing 3D scenes and 3D assets in VR or AR. With the mobile client, users who have registered in the living lab environment can answer traditional surveys or enlist in activities. Moreover, users can launch the AR client from the mobile client. The AR client is integrated into the mobile client for providing location-based AR functions for the mobile user [6]. This solution provides users an opportunity to participate in the development of products and services by giving location-based comments and feedback. The VR client allows users to experience accurate $3 \mathrm{D}$ representations of target areas. It provides a method for collecting UX and feedback from users who are immersed in VR and observing 3D content. The feedback can be collected while users explore various types of VEs e.g. learning environments, in both indoor and outdoor, realistic or fantastic even abstract environments. Users can communicate their experience by answering survey questions while exploring. The current tool supports VR questionnaires that are based on downtown area of Oulu, Finland, as well as campus of the University of Oulu that are modelled as mirror 3DVEs.

\section{Study Setup}

The focus of our study was to investigate, how participants experienced the VR and AR clients as well as how important they perceive it that they can answer to questions while experiencing a 3D environment. They answered questionnaires embedded in VR and AR clients. The use case for our evaluation was topical for the 
local community: the digitalization of the contents of a local zoological museum exhibition that has been recently closed.
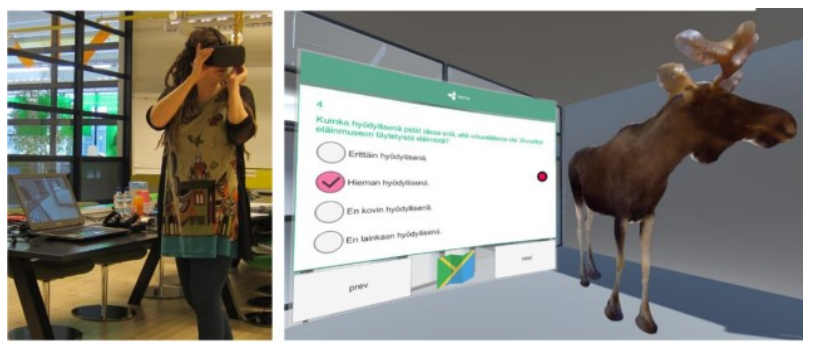

Figure 1: A study participant (left) and what she sees (right).

We had 14 participants in our study. Before attending the test session, participants filled a short background questionnaire online. Half of the participants used the VR client first (Figure 1) and AR client second, half vice versa. The users were recruited from the living lab's own user community. None of the participants had previous experience with either client. Participants' ages were between 26-59 years, with the average age of 36. Six participants were female. Duration of each test was approximately one hour. Users were interviewed and observed during the sessions. All sessions were also video recorded. In addition to interviews and observation, we collected material with a questionnaire on fivepoint Likert scale and with adjective cards selection method [9].

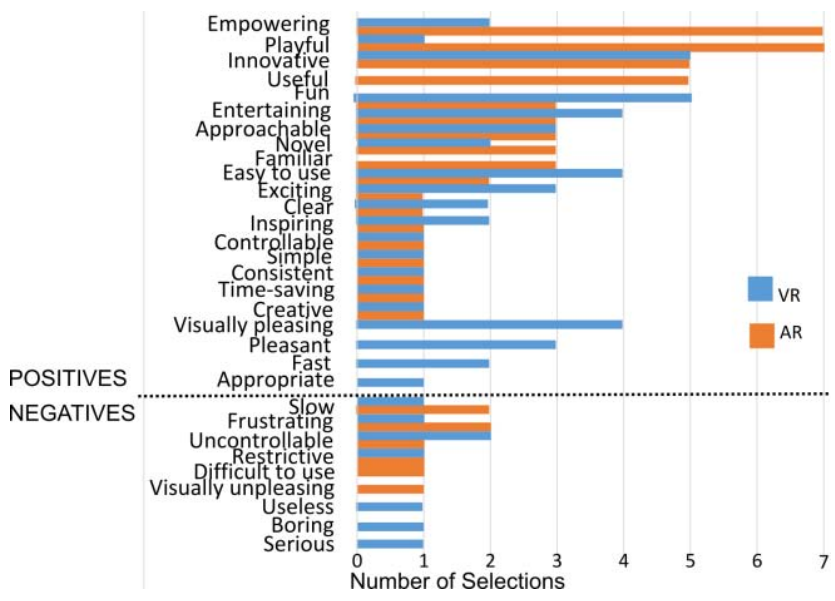

Figure 2: Adjectives for the VR method and for comparison AR. Each participant selected $4 / 42$ adjectives from a shuffled selection of 21 positives and 21 negatives.

\section{Results}

According to results, participants' experiences towards VR method were mainly positive (Figure 2 ). The most selected adjectives were Fun (5) and Innovative (5). Selected negative adjectives suggest usability issues or lack in visual quality. The visual quality of the virtual environment was reasonable, but not very detailed since performance issues in Web VR had to be considered. The most prominent adjectives selected to describe the AR experience were empowering (7), and playful (7). The fate of the zoological museum collection was an acute topic for participants. They did not perceive VR as a game, but an honest attempt to utilize digitalized contents of the zoological museum in VR. Also, participants commented that it was interesting for them that they were in the same place physically and virtually. The perception of AR was related to the playful nature of the experience; the users hoped for wider exploration of the physical campus for finding more hidden content.

\section{Conclusions}

Living labs can benefit from using AR and VR technologies. Both can provide comprehensive ways of visualizing change. VR provides users an interactive immersive environment, where user feedback can be collected by responding to surveys while experiencing accurate $3 \mathrm{D}$ representations of the target areas (whether existing or historical/imaginary). Similarly, AR technologies can project change directly on top of existing locations.

According to our findings, both VR and AR clients were experienced positively, while they do provide different kinds of UX. This is important to acknowledge when combining technologies or aiming to design a system for collecting user feedback. VR was perceived as innovative, easy to use, entertaining and fun. Participants considered important that they can experience the environment related to the survey while answering the survey. In comparison, the AR client was experienced empowering and playful. Our users envisioned AR more suitable for creating playful contents for exploration in the physical world, whereas VR was perceived more suitable for providing serious, pleasant and entertaining experiences.

\section{ACKNOWLEDGEMENTS}

This work has been supported by the Open Innovation Platforms spearhead project (A70202) and the Open City Model as Open Innovation Platform project (A71143) funded by the ERDF, the COMBAT project (293389) and Reboot IoT Factory project. Warm thanks to our test users.

\section{REFERENCES}

[1] Max Allen, Holger Regenbrecht, and Abbott, "Smart-phone augmented reality for public participation in urban planning", Proceedings of the 23rd Australian computer-human interaction conference, Canberra, Australia, pp. 11-20, 2011.

[2] Christian Dibbern, Manuela Uhr, Dennis Krupke, and Frank Steinicke. 2018. "Can WebVR further the adoption of Virtual Reality?", Mensch und Computer 2018-Usability Professionals. Dresden, Germany, 2018.

[3] Verónica Gutiérrez, Jose A. Galache, Luis Sánchez, Luis Muñoz, José M. Hernández-Muñoz, Joao Fernandes, and Mirko Presser. "SmartSantander: Internet of things research and innovation through citizen participation", The Future Internet Assembly, Springer, US, 173-186. 2013.

[4] Victoria Karaseva, Ahmed Seffah, and Jari Porras. "A social-mediabased living lab: an incubator for human-centric software engineering and innovation", Proceedings of the 2015 International Conference on Software and System Process, Tallinn, Estonia, 194-198. 2015.

[5] Michael D. McNeese."How video informs cognitive systems engineering: making experience count", Cognition, Technology \& Work, Springer, US, pp. 186-196, 2004.

[6] Arttu Niemelä. "Mobile augmented reality client for citizen participation", Master's thesis, Oulu, Finland, 2018.

[7] Jo Pierson and Bram Lievens, "Configuring living labs for a 'thick'understanding of innovation", Ethnographic Praxis in Industry Conference Proceedings, Redmond, WA, US, pp. 114-127, 2005.

[8] Stefan Reifinger, Florian Laquai, and Gerhard Rigoll. "Translation and rotation of virtual objects in Augmented Reality: A comparison of interaction devices. Systems, Man and Cybernetics", IEEE International Conference on SMC, Singapore, pp. 2448-2453, 2008.

[9] Meiju Sunnari, Leena Arhippainen, Minna Pakanen, and Seamus Hickey. "Studying User Experiences of Autostereoscopic 3D Menu on Touch Screen Mobile Device". Proceedings of the 25th Annual Conference of the Australian Computer-Human Interaction Special Group (OZCHI), Melbourne, Australia, 558-561, 2012. 